\title{
Analytical calculations of the solid angle subtended by a circular detector at linear sources
}

\author{
Mahmoud I. Abbas
}

\begin{abstract}
Knowledge of the geometrical solid angle is essential in all absolute measurements of the strengths of radioactive materials and to calibrate detectors. A direct mathematical formalism for the determination of the geometrical solid angle and the geometrical efficiency of a circular detector and an arbitrarily positioned line source is deduced. The results have been compared with previous computational treatments. The comparison shows a very satisfactory agreement in all cases.
\end{abstract}

Keywords: Geometrical solid angle; Geometrical ef?ciency; Linear sources; Circular detector.

\section{Introduction}

The geometrical solid angle $\Omega$ is widely used during absolute methods to calibrate detectors or to determine the activity of a radioactive source. This parameter describes the angular extent of particle or ray emitted by a point source and collected by the detector system. The ideal detector would surround an isotropically radiating point source and has a geometrical solid angle of $4 \pi$ steradians. Due to the practical limitations of sample shape and instrumentation access, as well as the physical geometry of the detector reaching this level of geometrical efficiency (i.e., $4 \pi=100 \%$ ), is unrealizable. Nevertheless, the specification and use of geometrical solid angle as a qualifying parameter which can be used to calculate the benefits of a detector configuration, rather than its physical size, is an important distinction. This is most important when assessing various geometries as physically larger detectors do not always correlate with greater geometrical solid angles and thus more efficient and statistically significant data collection or greater sensitivity capabilities. Conway (2010) has been reported recently [1], the treatments of the geometrical solid angle subtended by a circular detector at line source. He used a new axisymmetric radiation vector potential method

Manuscript received November 11, 2018; accepted May 25, 2018.

Mahmoud I. Abbas is with the Physics Department, Faculty of Science, Alexandria University, 21511 Alexandria, Egypt 
to solve the geometrical efficiency of a line source and a circular disk detector when the line source is parallel to the detector axis. Recently, Selim and Abbas [2-15] calculated the geometrical, total and full-energy peak efficiencies for any source-detector configuration using spherical coordinates system. In this paper using the previous approach, we present a direct mathematical method to calculate the geometrical solid angle subtended by a cylindrical detector at arbitrarily positioned line sources. The arrangement of this paper is as follows. Section 2 presents direct mathematical formulae for the geometrical solid angle, and consequently the geometrical ef?ciency $\left(\varepsilon_{g}=\Omega / 4 \pi\right)$, in the case of an arbitrarily positioned line source. Section 3 contains the validation of the present method. Conclusions are presented in Section 4.

\section{Mathematical viewpoint}

In the present work we derive a statistical integral formulation for the geometrical solid angle subtended by a circular detector of radius $\mathrm{R}$ at an arbitrarily positioned isotropic radiating point source, with lateral distance $\rho$, by using a spherical coordinate technique. The geometrical solid angle is given by [2]:

$$
\Omega=2 \pi-\int_{0}^{2 \pi} g(\rho, \xi, h) d \xi
$$

where,

$$
g(\rho, \xi, h)=\frac{h\left(h^{2}+\rho^{2}+R \rho \sin \xi\right)}{\left(h^{2}+\rho^{2} \cos ^{2} \xi\right)\left(h^{2}+R^{2}+\rho^{2}+2 R \rho \sin \xi\right)^{\frac{1}{2}}}
$$

The geometrical notations of $h, R$ and $\rho$ are as shown in Fig. 1.

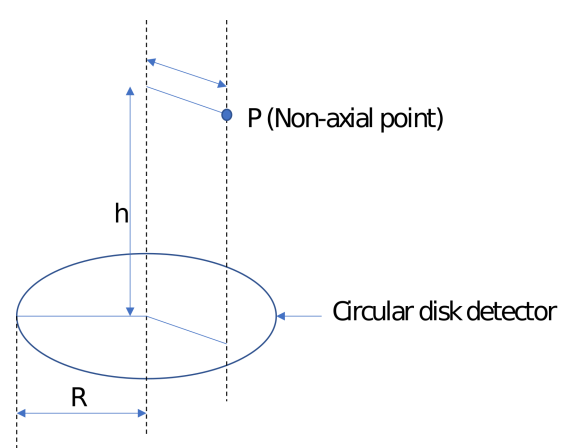

Fig. 1.

The work described below involves the use of direct analytical formulae for the computation of the geometrical solid angle subtended by a circular detector at linear sources assumed to be located along and parallel to its axis. 


\subsection{Line source assumed to be located along the axis of a circular detector}

The geometrical solid angle subtended by a circular detector of radius $\mathrm{R}$ and a line source assumed to be located along the axis of a circular detector is shown in Fig 2, is given by:

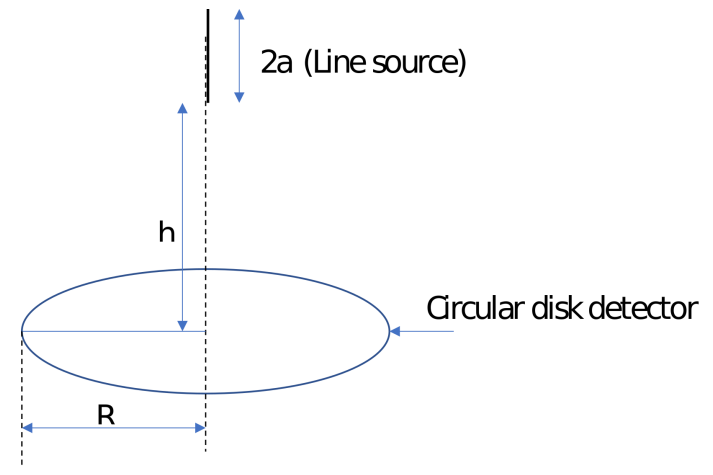

Fig. 2.

$$
\begin{gathered}
\Omega=\frac{1}{2 a} \int_{h-a}^{h+a}\left(2 \pi-\int_{0}^{2 \pi} g(0, \xi, h) d \xi\right) d h \\
\varepsilon_{g}=\frac{\Omega}{4 \pi} \\
\varepsilon_{g}=\frac{1}{4 \pi \cdot 2 a} \int_{h-a}^{h+a}\left(2 \pi-\int_{0}^{2 \pi} g(0, \xi, h) d \xi\right) d h \\
\varepsilon_{g}=\frac{1}{4 a} \int_{h-a}^{h+a}\left(1-\frac{h}{\sqrt{h^{2}+R^{2}}}\right) d h
\end{gathered}
$$

where, $g(\rho, \xi, h)$ is as identified before in equation (2).

\subsection{Line source assumed to be located parallel to the axis of a circular detector}

The geometrical solid angle subtended by a circular detector of radius $\mathrm{R}$ and a line source assumed to be located parallel to the axis of a circular detector is shown in Fig 3.

$$
\varepsilon_{g}=\frac{1}{4 \pi \cdot 2 a} \int_{h-a}^{h+a}\left(2 \pi-\int_{0}^{2 \pi} g(\rho, \xi, h) d \xi\right) d h
$$

where, $g(\rho, \xi, h)$ is as identified before in equation (2). Setting the lateral distance ? $=0$ in the previous equations, we obtain the geometrical efficiency of a circular detector (with radius R) and a line source assumed to be located along the axis of a circular detector (equation 6). 


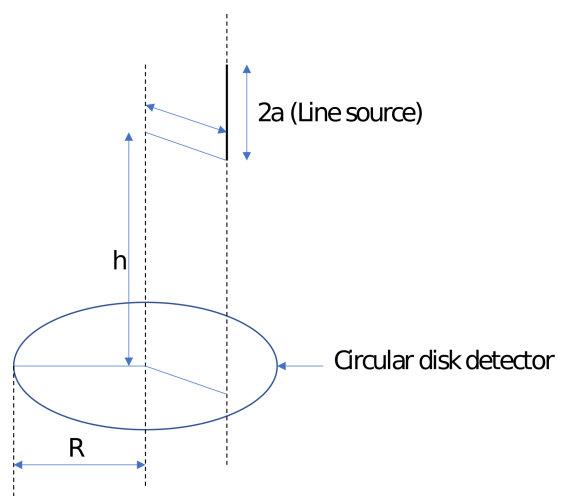

Fig. 3.

\section{Validation of the present method}

Systematic calculations of the geometrical efficiency of a circular detector of radius $\mathrm{R}$ and a line source located along and parallel to the axis of a circular detector is were calculated and tabulated in tables 1 and 2, respectively. The percentage deviations between calculated ef?ciency values (using the present formulae and that published by Conway, 2010 [1]) are less than $\left(10^{-10} \%\right)$. The percentage deviation is given by:

$$
\Delta \%=\frac{\varepsilon_{\text {presentwork }}-\varepsilon_{\text {Conway }}}{\varepsilon_{\text {presentwork }}} \times 100 \%
$$

Table 1. Geometrical efficiency $\varepsilon_{g}$ for a circular detector of radius $\mathrm{R}$ and a linear source of length 2a. The source lies along the detector axis with its center at a distance $\mathrm{h}$ from the detector. Distances are in arbitrary units

\begin{tabular}{|l|l|l|l|l|}
\hline \multirow{2}{*}{$\mathrm{R}$} & \multirow{2}{*}{$\mathrm{h}$} & $\mathrm{a}$ & \multicolumn{2}{|c|}{ Geometrical efficiency $\left(\varepsilon_{g}\right)$} \\
\cline { 4 - 5 } & & & Conway [2] & Present work \\
\hline 10 & 5 & 3 & 0.282649212693323 & 0.282649212693323 \\
\hline 10 & 5 & 5 & 0.292893218813452 & 0.292893218813452 \\
\hline $10^{5}$ & 5 & 5 & 0.499975000000062 & 0.499975000000062 \\
\hline 10 & 1 & 1 & 0.450490243203608 & 0.450490243203608 \\
\hline 10 & 0.1 & 0.1 & 0.495000499900025 & 0.495000499900025 \\
\hline 10 & 10 & 2 & 0.148218640381549 & 0.148218640381549 \\
\hline 10 & 100 & 2 & $2.48238070579414 \times 10^{-3}$ & $2.48238070579414 \times 10^{-3}$ \\
\hline 10 & 100 & 100 & $2.43753901374804 \times 10^{-2}$ & $2.43753901374804 \times 10^{-2}$ \\
\hline 5 & 5 & 5 & 0.190983005625053 & 0.190983005625053 \\
\hline 5 & 5 & 4 & 0.175211835787862 & 0.175211835787862 \\
\hline
\end{tabular}


Table 2. Geometrical efficiency $\varepsilon_{g}$ for a circular detector of radius $\mathrm{R}$ and a linear source of length $2 \mathrm{a}$. The source and detector axes are parallel and separated by a lateral distance $\rho$. The center of the source is an axial distance $\mathrm{h}$ from the detector plane. Distances are in arbitrary units.

\begin{tabular}{|l|l|l|l|l|l|}
\hline \multirow{2}{*}{$\mathrm{R}$} & \multirow{2}{*}{$\rho$} & $\mathrm{h}$ & $\mathrm{a}$ & \multicolumn{2}{|c}{ Geometrical efficiency $\left(\boldsymbol{\varepsilon}_{g}\right)$} \\
\cline { 5 - 6 } & & & & Conway $[1]$ & Present work \\
\hline 10 & 1 & 6 & 1 & 0.2424075268155220 & 0.2424075268155218 \\
\hline 10 & 1 & 6 & 2 & 0.2444939236648650 & 0.2444939236648647 \\
\hline 10 & 1 & 6 & 3 & 0.2479120907431220 & 0.2479120907431219 \\
\hline 10 & 1 & 6 & 4 & 0.2525702335275070 & 0.2525702335275068 \\
\hline 10 & 1 & 6 & 5 & 0.2583365907829720 & 0.2583365907829719 \\
\hline 10 & 1 & 6 & 6 & 0.2650417921134300 & 0.2650417921134298 \\
\hline 20 & 1 & 6 & 6 & 0.3613152934378430 & 0.3613152934378427 \\
\hline 50 & 1 & 6 & 6 & 0.4408231864430030 & 0.4408231864430028 \\
\hline $10^{2}$ & 1 & 6 & 6 & 0.4701050189567020 & 0.4701050189567020 \\
\hline $10^{3}$ & 1 & 6 & 6 & 0.4970001057426270 & 0.4970001057426270 \\
\hline $10^{6}$ & 1 & 6 & 6 & 0.4999970000000000 & 0.4999970000000000 \\
\hline 5 & 1 & 5 & 4 & 0.1726042018147730 & 0.1726042018147727 \\
\hline 5 & 2 & 5 & 4 & 0.1645441216554290 & 0.1645441216554288 \\
\hline 5 & 3 & 5 & 4 & 0.1502632976034040 & 0.1502632976034037 \\
\hline 5 & 4 & 5 & 4 & 0.1283998422373930 & 0.1283998422373929 \\
\hline 5 & 5 & 5 & 4 & 0.0988096991450620 & 0.0988096991450618 \\
\hline 5 & 6 & 5 & 4 & 0.0706900253117640 & 0.0706900253117636 \\
\hline 5 & 7 & 5 & 4 & 0.0512352434451088 & 0.0512352434451085 \\
\hline 5 & 8 & 5 & 4 & 0.0381704509705044 & 0.0381704509705041 \\
\hline 5 & 9 & 5 & 4 & 0.0291077849249144 & 0.0291077849249142 \\
\hline 5 & 10 & 5 & 4 & 0.0226308012432528 & 0.0226308012432526 \\
\hline 20 & 5 & 1 & 1 & 0.4738338789017140 & 0.4738338789017137 \\
\hline 20 & 5 & 2 & 1 & 0.4479010451301750 & 0.4479010451301748 \\
\hline 20 & 5 & 3 & 1 & 0.4224234986937620 & 0.4224234986937619 \\
\hline 20 & 5 & 4 & 1 & 0.3976019974352420 & 0.3976019974352418 \\
\hline 20 & 5 & 5 & 1 & 0.3736084804781520 & 0.3736084804781518 \\
\hline 20 & 5 & 10 & 1 & 0.2697132766757590 & 0.2697132766757587 \\
\hline 20 & 5 & 20 & 1 & 0.1424329648079320 & 0.1424329648079317 \\
\hline 20 & 5 & 40 & 1 & 0.0519778159212070 & 0.0519778159212052 \\
\hline
\end{tabular}




\section{Conclusions}

In this paper, it becomes easier using a compact mathematical expression to calculate the geometrical efficiency of a circular detector of radius $\mathrm{R}$ and a line source located along and parallel to the axis of a circular detector. The agreement between the results calculated in this work and the published values is excellent; the percentage deviation was vanished $(0.00 \%)$. This means that the present approach is efficient and sufficiently powerful to evaluate the geometrical efficiency of circular detectors.

\section{References}

[1] J.T. CONWAY, Geometric efficiency for a circular detector and a linear source of arbitrary orientation and position, Nuclear Instruments and Methods in Physics Research A622, (2010) 555-566.

[2] Y.S. Selim, M.I. Abbas, Source-detector geometrical ef?ciency, Radiat. Phys. Chem. 44, (1994) $1-4$.

[3] M.I. ABBAS, A direct mathematical method to calculate the efficiencies of a parallelepiped detector for an arbitrarily positioned point source, J. Radiat. Phys. Chem.60,(2001) 3.

[4] M.I. АввAS, HPGe detector photopeak efficiency calculation including self-absorption and coincidence corrections for Marinilli beaker sources using compact analytical expressions, Radiat. Phys. Chem. 54, (2010) 761.

[5] M.I. Abbas, Y.S. Selim, Calculation of relative full-energy peak efficiencies of well-type detectors, Nucl. Instrum.MethodsPhys. Res. A480, (2002) 651.

[6] M.I. Abbas, Y.S. Selim, S. NAFEE, A simple mathematical method to determine the efficiencies of log-conical detectors, Radiat. Phys. Chem. 75 (7), (2006) 729-736.

[7] Y.S. Selim, M.I. AbBas, M. Fawzy, Analytical calculation of the efficiencies of gamma scintillators. Part I: Total efficiency for coaxial disk sources, Radiat. Phys. Chem. 53 (6), (1998) 589-592.

[8] Y.S. SElim, M.I. ABBAS, Analytical calculations of gamma scintillators efficiencies. II: total efficiency for wide co-axial disk sources, Radiat. Phys. Chem. 53, (2000) 15-19.

[9] M.I. AbBas, S. NoureddeEn, Analytical expression to calculate total and full-energy peak efficiencies for cylindrical phoswich and lanthanum bromide scintillation detector, Rad. Measur, 46, (2011) 440-445.

[10] M.I. ABBAS, Analytical approach to calculate the efficiency of 4? NaI(Tl) gamma-ray detectors for extended sources, Nuclear Instruments \& Methods in Physics Research - A615, (2010) 48-52.

[11] M.I. AbBas, A new analytical method to calibrate cylindrical phoswich and $\mathrm{LaBr} 3$ (Ce) scintillation detectors, Nuclear Instruments \& Methods in Physics Research - A621, (2010) 413-418.

[12] M.I. ABBAS, Analytical formulae for borehole scintillation detectors efficiency calibration, Nuclear Instruments \& Methods in Physics Research - A622, (2010) 171-175. 
[13] M.I. Abbas, S. Hammoud, T. Ibrahim, M. SAKR, Analytical formulae to calculate the solid angle subtended at an arbitrarily positioned point source by an elliptical radiation detector, Nuclear Instruments \& Methods in Physics Research - A771, (2015) 121.

[14] M.I. Abbas, S. Hammoud, T. Ibrahim, M. SAKR, Analytical formulae to calculate the total efficiency of an arbitrarily positioned point source by an elliptical cylindrical detector,Journal of Nuclear Sciences, Vol. 2, No. 2, (2015) 11-22.

[15] M.I. Abbas, M.S. Badawi, I.N. Ruskov, A.M. El-Khatib, D.N. Grozdanov, A. A. Thabet, Yu.N. Kopatch, M.M. Gouda, V.R. SkoY, Calibration of a single hexagonal NaI(Tl) detector using a new numerical method based on the efficiency transfer method, Nuclear Instruments \& Methods in Physics Research - A771, (2015) 110. 